\title{
ON CONSTRUCTING NEARLY DECOMPOSABLE MATRICES
}

\section{J. HARTFIEL}

Abstract. In this paper we consider the construction of nearly decomposable matrices from nearly decomposable matrices of smaller dimension. In particular, if $A_{1}, A_{2}, \cdots, A_{\text {, are nearly }}$ decomposable matrices we consider the problem of finding matrices $E_{1}, E_{2}, \cdots, E_{8}$ each with exactly one nonzero entry so that

$$
\left(\begin{array}{ccccc}
A_{1} & 0 & \cdots & 0 & E_{1} \\
E_{2} & A_{2} & \cdots & 0 & 0 \\
\cdots & \cdots & \cdots & \cdots & \cdots \\
0 & 0 & \cdots & E_{8} & A_{8}
\end{array}\right)
$$

is nearly decomposable.

Introduction and notation. In [6] Sinkhorn and Knopp introduced the notion of nearly decomposable matrices. These matrices were used to solve a conjecture of Marshall Hall in [5], and to find lower bounds for the permanent function on certain classes of $(0,1)$ matrices in [4]. The structure of these matrices is given below.

Theorem I. Suppose $A$ is an $n \times n$ nonnegative matrix, $n>1$. If $A$ is nearly decomposable then there exist permulation matrices $P$ and $Q$ and an integer $s>1$ so that

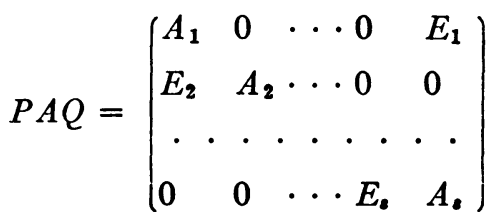

where each $A_{k}$ is nearly decomposable and $E_{1}, E_{2}, \cdots, E_{8}$ have exactly one nonzero entry [6].

In [2] it is shown that $A_{1}, A_{2}, \cdots, A_{8-1}$ may be assumed to be of dimension one.

In this paper we consider ways in which nearly decomposable matrices may be constructed from nearly decomposable matrices of lower dimension. In particular, if $A_{1}, A_{2}, \cdots, A_{2}$ are nearly decomposable matrices we wish to find where positive numbers may be placed in the $E_{k}$ 's so that

Received by the editors October 3, 1969.

AMS 1969 subject classifications. Primary 1530; Secondary 1548.

Key words and phrases. (0, 1)-matrices, construction, canonical form. 


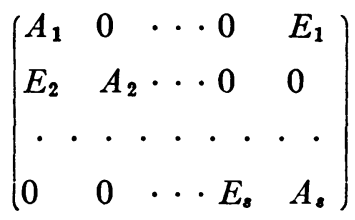

is nearly decomposable.

Of course, if we arbitrarily place a positive number in each $E_{k}$ then the resulting matrix is fully indecomposable by the following theorem.

Theorem II. Suppose $A$ is an $n \times n$ nonnegative matrix of the form

$$
\left(\begin{array}{ccccc}
A_{1} & 0 & \cdots & 0 & E_{1} \\
E_{2} & A_{2} & \cdots & 0 & 0 \\
\cdot & \cdot & \cdots & \cdot & \cdot \\
0 & 0 & \cdots & E_{s} & A_{\imath}
\end{array}\right)
$$

where $s>1$ and for $k=1,2, \cdots, s ; A_{k}$ is fully indecomposable and $E_{k}$ has exactly one positive entry. Then $A$ is fully indecomposable $[6, p$. 69 ].

This does not imply however that the resulting matrix is nearly decomposable. In fact, examples can easily be found where this is not the case.

We include the following definitions and notation.

A nonnegative $n$-square matrix $A, n>1$, is partly decomposable if there exist permutation matrices $P$ and $Q$ so that $P A Q=\left(\begin{array}{ll}X & 0 \\ Y & Z\end{array}\right)$ where $X$ and $Z$ are square. Otherwise $A$ is fully indecomposable. By convention a $1 \times 1$ matrix is fully indecomposable if and only if its single entry is positive. Let $E_{i j}$ be an $n$-square $(0,1)$-matrix having a one in the $i j$ th position and 0 's elsewhere. If $A=\left(a_{i j}\right)$ is fully indecomposable and for each $a_{i j} \neq 0$ we have $A-a_{i j} E_{i j}$ partly decomposable, then we say that $A$ is nearly decomposable.

If $\sigma$ is a permutation of $1,2, \cdots, n$ and $a_{1 \sigma(1)}, \cdots, a_{n \sigma(n)}$ are all positive entries of $A$ then we call $a_{1 \sigma(1)}, \cdots, a_{n \sigma(n)}$ a positive diagonal of $A$. Each $a_{k \sigma(k)}$ is said to lie on the positive diagonal. If each $a_{i j} \neq 0$ in $A$ lies on some positive diagonal of $A$ then we say that $A$ has total support. By using the Frobenius-Konig Theorem [3, p. 97 it is easily shown that fully indecomposable implies total support.

In order to simplify much of the following we denote by $A=\left(a_{i j}\right)$ an $n \times n$ nearly decomposable $(0,1)$-matrix in the $\left(A_{k}, E_{k}\right)$ form of Theorem I. Further suppose that the one in $E_{k}$ is in the $\left(i_{k}, j_{k}\right)$ position of $A$. 
Results. In order to construct nearly decomposable matrices from nearly decomposable matrices of lower dimension we introduce the following definition.

Definition. Suppose $\left(p_{1}, q_{1}\right), \cdots,\left(p_{r}, q_{r}\right)$ are 0 positions in $A$. Let $B$ denote the $n \times n(0,1)$-matrix with 1 's only in the $\left(p_{1}, q_{1}\right), \cdots$, $\left(p_{r}, q_{r}\right)$ positions. Suppose $A+B$ has the property that if any 1 in $A$ is replaced by a 0 yielding $\bar{A}$ we have that $\bar{A}+B$ is partly decomposable. We then call $\left\{\left(p_{1}, q_{1}\right), \cdots,\left(p_{r}, q_{r}\right)\right\}$ a tie set for $A$. A position $(p, q)$ for which $\{(p, q)\}$ is a tie set is called a tie point for $A$.

EXAMPLE.

(1) The tie set is empty.

$\left(\begin{array}{l}11 \\ 11\end{array}\right) \quad$ The tie set is empty.
$\left(\begin{array}{l}110 \\ 011 \\ 101\end{array}\right) \quad\{(1,3)\}\{(2,1)\}\{(3,2)\}$

It may also be noted that a subset of a tie set is itself a tie set.

We now show how to find tie sets. For this we need the following lemma.

Lemma. Suppose $B$ is an $n \times n$ fully indecomposable $(0,1)$-matrix, $n>1$. If some 1 in $B$ is replaced by $a 0$, the resulting matrix $\bar{B}$ is partly decomposable if and only if $\bar{B}$ does not have total support.

Proof. If $\bar{B}$ is partly decomposable then there exist permutation matrices $P$ and $Q$ so that

$$
P \bar{B} Q=\left(\begin{array}{ll}
X & 0 \\
Y & Z
\end{array}\right)
$$

where $X$ and $Z$ are square. Since $B$ was fully indecomposable, $Y$ is not 0 . Hence $\bar{B}$ does not have total support.

Conversely if $\bar{B}$ does not have total support, then it is partly decomposable since any fully indecomposable matrix has total support.

TheOREM 1. If $A_{k}$ is not $1 \times 1$ then the $\left(i_{k}, j_{k+1}\right)$ position is a 0 position of $A ; k, k+1 \bmod s .\left\{\left(i_{k}, j_{k+1}\right) \mid A_{k}\right.$ is not $\left.1 \times 1\right\}$ is a tie set for $A$. Further the position $\left(i_{k}, j_{k+1}\right)$ is a tie point for $A_{k}$ for each $A_{k}$ not $1 \times 1$.

Proof. Suppose there is a 1 in the $\left(i_{k}, j_{k+1}\right)$ position of $A, A_{k}$ not $1 \times 1$. Replace this 1 in $A$ by 0 calling this matrix $\bar{A}$ and having $\left(\bar{A}_{l}, E_{l}\right)$ form: 


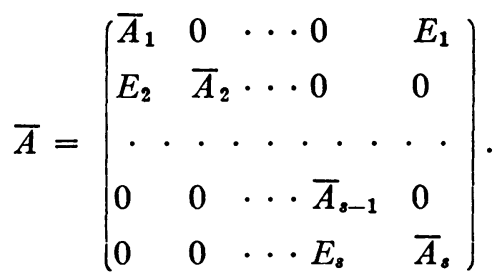

Now $\bar{A}_{k}$ and $\bar{A}$ do not have total support since $A_{k}$ and $A$ are nearly decomposable. Since $A_{k}$ has at least two 1's in each row and column and has total support there exists at least one positive diagonal in $\bar{A}_{k}$. Therefore if $l \neq k$, then every 1 in $\bar{A}_{l}$ is on a positive diagonal of $\bar{A}$. The 1's in the $E_{p}$ 's $(p=1,2, \cdots, s)$ are also still on a positive diagonal of $\bar{A}$. Pick some 1 in $\bar{A}_{k}$. If it is on a positive diagonal in $\bar{A}_{k}$ then it is on a positive diagonal in the $\bar{A}_{l}$ 's of $\bar{A}$. If it is not on a positive diagonal of $\bar{A}_{k}$, then it is on a positive diagonal of $A_{k}$, and hence on a positive diagonal with the 1's in the $E_{p}$ 's in $\bar{A}$. But now $\bar{A}$ has total support. This gives us a contradiction. Hence it follows that there must have been a 0 in the $\left(i_{k}, j_{k+1}\right)$ position of $A$.

To show $\left\{\left(i_{k}, j_{k+1}\right) \mid A_{k}\right.$ not $\left.1 \times 1\right\}$ is a tie set for $A$ we proceed as follows. Denote by $B$ the $\mathrm{n} \times n(0,1)$-matrix with 1 's in the $\left(i_{k}, j_{k+1}\right)$ positions and 0's elsewhere, $A_{k}$ not $1 \times 1$. Hence

$$
B=\left(\begin{array}{llll}
B_{1} & 0 & \cdots & 0 \\
0 & B_{2} & \cdots & 0 \\
\cdot & \cdot & & \cdot \\
\cdot & \cdot & & \cdot \\
0 & 0 & \cdots & B_{8}
\end{array}\right)
$$

where each $B_{k}$ is square and of the same dimension as $A_{k}$. Now $A+B$ is an $n \times n(0,1)$-matrix. Replace some 1 in $A$ by 0 calling this matrix $\bar{A}$ having $\left(\bar{A}_{l}, \bar{E}_{l}\right)$ form. We now show $\bar{A}+B$ is partly decomposable.

If the 1 replaced by 0 in $A$ was in some $E_{k}$, or in some $A_{k}$ which was $1 \times 1$, then $\bar{A}+B$ is clearly partly decomposable. Therefore we suppose the 1 replaced by 0 in $A$ was in $A_{r}$ for some $r$ so that $A_{r}$ is not $1 \times 1$. For this, suppose $\bar{A}+B$ is fully indecomposable and hence has total support. Now $\bar{A}_{r}$ still has at least one positive diagonal. Hence each 1 in $\bar{A}_{k}, k \neq r$, is on a positive diagonal in $\bar{A}$. Since $\bar{A}+B$ has total support, the 1 's in the $E_{k}$ 's are on a positive diagonal in $\bar{A}$. Pick any 1 in $\bar{A}_{r}$. If it is on a positive diagonal with the 1 in the $\left(i_{r}, j_{r+1}\right)$ position in $\bar{A}_{r}+B_{r}$, then it is on a positive diagonal with the 1 's in the $E_{k}$ 's in $\bar{A}$. If it is not on a positive diagonal with the 1 in the $\left(i_{r}, j_{r+1}\right)$ 
position in $\bar{A}_{r}+B_{r}$, then it is on a positive diagonal in the $\bar{A}_{k}$ 's of $\bar{A}$. Hence we have shown that $\bar{A}$ has total support which is a contradiction to $A$ being nearly decomposable. Therefore $\left\{\left(i_{k}, j_{k+1}\right) \mid A_{k}\right.$ is not $1 \times 1\}$ is a tie set for $A$.

As above, it can be shown that if $\bar{A}_{r}+B_{r}$ is fully indecomposable, then so is $\bar{A}$. Hence we see that $\bar{A}_{r}+B_{r}$ is partly decomposable and hence $\left(i_{r}, j_{r+1}\right)$ is a tie point for $A_{r}$.

Corollary 1. Suppose $A_{1}$ in $A$ is not $1 \times 1$. If a 1 in $A_{1}$ is replaced by 0 , there results a matrix $\bar{A}$, and there exist permutation matrices? $P_{a}^{?}$ and $Q$ so that

$$
P \bar{A} Q=\left(\begin{array}{cc|cccc}
X & 0 & 0 & \cdots & 0 & 0 \\
Y & Z & 0 & \cdots & 0 & F \\
\hdashline E & A_{2} & \cdots & 0 & 0 \\
\cdots & \cdots & \cdots & \cdots & \cdots \\
0 & 0 & \cdots & A_{\&-1} & 0 \\
0 & 0 & \cdots & E_{\bullet} & A_{\bullet}
\end{array}\right)
$$

or

$$
P \bar{A} Q=\left(\begin{array}{cc|cccc}
X & 0 & 0 & \cdots & 0 & E \\
Y & Z & & & & \\
\hline F & 0 & 0 & \cdots & 0 & 0 \\
\cdot & \cdots & \cdots & \cdots & \cdots & \cdot \\
0 & 0 & 0 & \cdots & A_{s-1} & 0 \\
0 & 0 & 0 & \cdots & E_{s} & A_{\bullet}
\end{array}\right)
$$

where $X$ and $Z$ are square, $E$ and $F$ have exactly one entry equal to 1 .

Proof. $\left(i_{1}, j_{2}\right)$ is a tie point for $A_{1}$. This determines the intersection of row $i_{1}$ and column $j_{2}$.

Corollary 2. No $A_{k}$ of $A$ is $\left(\begin{array}{l}1 \\ 1 \\ 1\end{array}\right)$.

Corollary 3. $A$ has a nonempty tie set if and only if $A$ is not $1 \times 1$ or $\left(\begin{array}{ll}1 & 1 \\ 1 & 1\end{array}\right)$.

Proof. If each $A_{k}$ of $A(k=1, \cdots, s)$ is $1 \times 1$ then any 0 position will do. If some $A_{k}$ of $A$ is not $1 \times 1$, then it cannot be $2 \times 2$ by the preceding corollary, hence $\left(i_{k}, j_{k+1}\right)$ will be a tie point for $A$.

We now show how to use tie sets in order to construct nearly decomposable matrices. 
TheOREM 2. If $A_{1}, A_{2}, \cdots, A_{8}$ are nearly decomposable matrices, no $A_{k}$ being $\left(\begin{array}{ll}1 & 1 \\ 1 & 1\end{array}\right)$ then there exists some $E_{k}$ 's so that

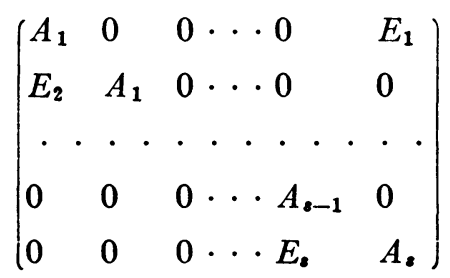

is nearly decomposable.

Proof. Each $A_{k}, A_{k}$ not $1 \times 1$, has a tie set. Take one point out of each tie set, say $\left(i_{k}, j_{k}\right)$. If $A_{k}$ is $1 \times 1$ then set $\left(i_{k}, j_{k}\right)=(1,1)$. Now if $A_{k}$ is $n_{k} \times n_{k}$ then consider the matrix $A, \sum n_{k} \times \sum n_{k}$ in $\left(A_{k}, E_{k}\right)$ form by putting the $A_{k}$ 's along the main diagonal and the 1 in the $E_{k}$ so that it is in row $i_{k}$ of $A_{k}$ and column $j_{k-1}$ of $A_{k-1} ; k, k-1 \bmod s$. We now show that $A$ is nearly decomposable.

By Theorem II, $A$ is fully indecomposable. To show $A$ is nearly decomposable we proceed as follows. If a 1 in some $E_{k}$ or some $A_{k}$ where $A_{k}$ is $1 \times 1$, is replaced by a 0 , yielding $\bar{A}$, then $\bar{A}$ is clearly partly decomposable. Further if a 1 in some $A_{k}, A_{k}$ not $1 \times 1$, is replaced by a 0 yielding $\bar{A}$, then since $\left(i_{k}, j_{k}\right)$ is a tie point for $A_{k}$ there exist permutation matrices $P$ and $Q$ so that $P A_{k} Q=\left\{\begin{array}{ll}X & 0 \\ Y & Z\end{array}\right\}$ where $X$ and $Z$ are square and the 0 in the $\left(i_{k}, j_{k}\right)$ position of $A_{k}$ is now in $X, Y$ or $Z$. Hence, since the tie point $\left(i_{k}, j_{k}\right)$ determines the intersection of row $i_{k}$ in $E_{k}$ and column $j_{k}$ in $E_{k+1}$ we see that $\bar{A}$ is partly decomposable, i.e., if $I_{l}$ denotes the $n_{l} \times n_{l}$ identity matrix then

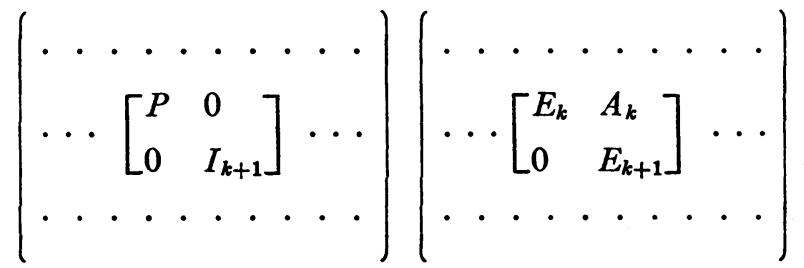

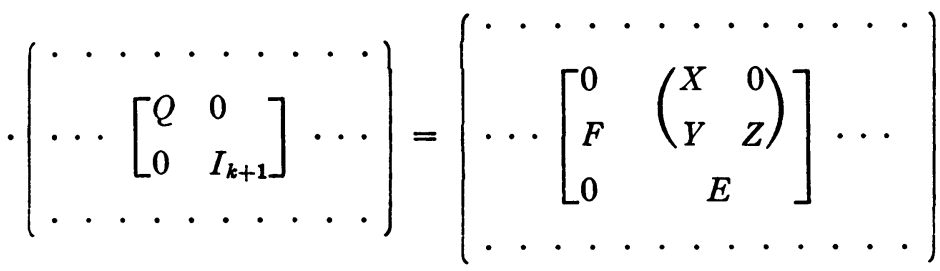




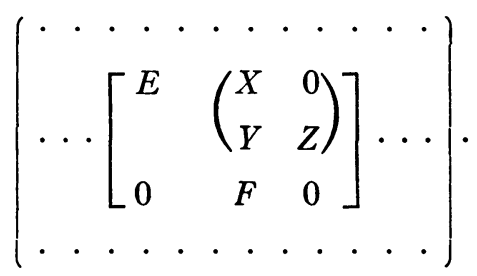

Corollary 1. If $A_{1}, A_{2}, \cdots, A_{s}$ are nearly decomposable, then there exist some $E_{k}$ 's so that

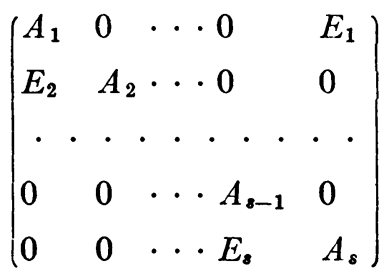

is nearly decomposable if and only if no $A_{k}$ is $\left(\begin{array}{ll}1 & 1 \\ 1 & 1\end{array}\right)$.

ACKNowledgement. I would like to express my thanks to the referee for his suggestions on the presentation of this paper.

\section{BIBLIOGRAPHY}

1. D. Hartfiel, On the structure of the certain matrix classes, Dissertation, Univ. of Houston, Houston, Tex., 1969.

2. - A simplified form for nearly reducible and nearly decomposable matrices, Proc. Amer. Math. Soc. 24 (1970), 388-393.

3. M. Marcus and H. Minc, $A$ survey of matrix theory and matrix inequalities, Allyn and Bacon, Boston, Mass., 1964. MR 29 \#112.

4. Henryk Minc, On lower bounds for permanents of $(0,1)$-matrices, Proc. Amer. Math. Soc. 22 (1969), 117-123.

5. Richard Sinkhorn, Concerning a conjecture of Marshall Hall, Proc. Amer. Math. Soc. 21 (1969), 192-201. MR 39 \#2780.

6. Richard Sinkhorn and Paul Knopp, Problems involving diagonal products in nonnegative matrices, Trans. Amer. Math. Soc. 136 (1969), 67-75.

Texas A\&M University, College Station, Texas 77843 